\footnotetext{
Revista Arbitrada Interdisciplinaria de Ciencias de la Salud. SALUD Y VIDA

Volumen 3. Número 5. Año 3. Enero - Junio 2019. Santa Ana de Coro, Venezuela.

Hecho el depósito de Ley: FA2016000010

ISSN: $2610-8038$

FUNDACIÓN KOINONIA (F.K). Santa Ana de Coro, Venezuela.
}

Maika Esther Bravo; Tibisay Lamus de Rodríguez

http://dx.doi.org/10.35381/s.v.v3i5.258

\title{
Atención integral al adulto mayor en Venezuela: un proceso de reflexión desde su recorrido institucional-legal
}

\section{Comprehensive care for the elderly in Venezuela: a process of reflection from its institutional-legal journey}

\author{
Maika Esther Bravo \\ maikabravo72@gmail.com \\ Universidad Nacional Experimental Francisco de Miranda \\ Venezuela \\ Tibisay Lamus de Rodríguez \\ lamus747@hotmail.com \\ Universidad Nacional Experimental Francisco de Miranda \\ Venezuela
}

Recibido: 13 de noviembre de 2018

Aprobado: 20 de diciembre de 2018

\section{RESUMEN}

El artículo tiene como objetivo presentar una revisión en torno a la atención integral ofrecida al adulto mayor desde el marco institucional-legal venezolano, lo cual ha permitido configurar una serie de fases por las cuales ha transitado las políticas públicas: de seguridad social, asistencia social, salud, como vías para ofrecer atención integral a personas la tercera edad. Esta construcción se ha convertido en un aporte para la ciencia gerontológica como ciencia destinada al estudio del proceso de envejecimiento y la vejez; así como para la carrera de gerontología al identificar, desde la visión de las atoras, elementos claves que han incidido en la atención de la persona mayor en Venezuela. Del mismo modo, en este proceso de revisión se han identificado la existencia de una imagen negativa sobre la vejez que son marco de acción en los programas sociales de carácter asistencialista implementados por los gobiernos en el devenir del tiempo. 


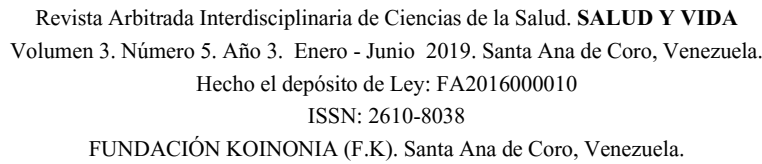

Maika Esther Bravo; Tibisay Lamus de Rodríguez

Descriptores: salud; gerontología; políticas de salud; envejecimiento demográfico; persona mayor

\section{SUMMARY}

The objective of the article is to present a review of the comprehensive care offered to the elderly from the Venezuelan institutional-legal framework, which has allowed to configure a series of phases through which public policies have gone through: social security, social assistance , health, as ways to offer comprehensive care to seniors. This construction has become a contribution to gerontological science as a science destined to the study of the aging process and old age; as well as for the career of gerontology when identifying, from the vision of the atoras, key elements that have influenced the attention of the elderly in Venezuela. In the same way, in this revision process, the existence of a negative image about old age has been identified as a framework of action in the welfare social programs implemented by governments in the course of time.

Descriptors:: health; gerontology; politic of health; aging demographic; elderly people

\section{INTRODUCCIÓN}

Uno de los aspectos que actualmente interesa a los Estados y su gobierno es el fenómeno de envejecimiento demográfico que ocurre cuando se registra un aumento considerable en la proporción de las personas de 60 años y más por encima de la población total; este fenómeno que inicialmente había sido experimentado por los países más desarrollados está convirtiéndose en un suceso global presente prácticamente en todo el mundo. (Sánchez: 2000) Este hecho ha sido el resultado del éxito en las políticas de salud, sociales y educativas que han incidido en las mejoras de las condiciones de vida de la población, favoreciendo por tanto, el incremento de la esperanza de vida al nacer, la disminución progresiva de las tasas de natalidad y mortalidad, factores éstos que incrementan de forma relativa la proporción de personas mayores.

Puede afirmarse entonces que, el envejecimiento demográfico ha de concebirse como un éxito para los Estados pero al mismo tiempo como un reto para las naciones al tener que garantizar a los adultos mayores que progresivamente van incrementándose en número, por un lado, el buen estado de salud asociada a la capacidad funcional, su participación, su seguridad social y por el otro, planificar políticas que permitan que más personas lleguen a la etapa de la vejez en forma saludable y activos. 


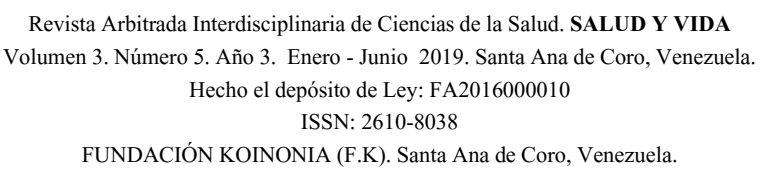

Maika Esther Bravo; Tibisay Lamus de Rodríguez

Este fenómeno ha sido de preocupación a nivel mundial a tal punto que en la Asamblea Mundial del Envejecimiento convocada por las Naciones Unidas en el año 1992, se instó a los autores de las políticas públicas a tomar en consideración que desde los años 60 la población mundial ha ido perdiendo sus características de población joven e hizo hincapié en que los adultos mayores y el proceso de envejecimiento individual y poblacional, aspectos estos que constituían preocupaciones internacionales como lo eran los niños y el desarrollo nacional. En respuesta a ese interés la misma organización designó el período entre 1975-2025 como la era del envejecimiento, período en el cual se han realizado avances en materia de instrumentos internacionales que favorezcan una vejez saludable y activa a nivel individual; así como se han realizado recomendaciones para que puedan propiciarse las oportunidades para que más personas puedan alcanzar esta etapa de la vida, sea respetada su dignidad, participación y seguridad en todos los entornos.

En el caso de Latinoamérica y el Caribe esta realidad no difiere puesto que los países de la región se han encaminado hacia etapas más avanzadas de la transición demográfica de forma paulatina pero ineludible. Esta realidad señala que, "De acuerdo con esta dinámica, la proporción de personas mayores de 60 años dentro de la población total se cuadruplicará entre 2000 y 2050, de modo que uno de cada cuatro latinoamericanos y caribeños será una persona mayor en el 2050"CELADE(2009:57). Este incremento progresivo de la población mayor ocasionará efectos de naturaleza multidimensional, desde las propias biografías de las personas, sus necesidades, el rol de la familia; así como las demandas de la población hacia el Estado en las esferas educacionales, económicas, sociales, medioambientales, recreativas y sobre todo sanitarias, puesto que el envejecimiento demográfico viene acompañado con un cambio significativo de los patrones epidemiológicos para lo cual los profesionales de la salud deberán estar preparados a atender.(Perez:1996)

Desde esta última perspectiva, las transformaciones relacionadas con el incremento de las proporciones absolutas o relativas de personas mayores en América Latina, traerá consigo el aumento en la incidencia de las enfermedades crónico-degenerativas y la 


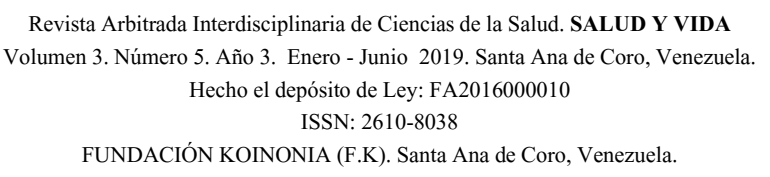

Maika Esther Bravo; Tibisay Lamus de Rodríguez

persistencia de algunas enfermedades de tipo transmisibles, características de los países en transición (CELADE:2006); en virtud que se ha comprobado una asociación entre la etapa de la vejez y la presencia de enfermedades como hipertensión arterial, diabetes, osteoartritis, entre otras, las cuales son el resultado de estilos de vida inadecuados instaurados desde edades tempranas. La complejidad de esta dinámica indica la necesidad de invertir tanto en las mejoras de los recursos tecnológicos y de infraestructura disponibles para tratar este tipo de enfermedades, como en la capacitación constante de los profesionales de la salud para atender una realidad altamente demandante de atención desde las acciones de prevención, el diagnóstico oportuno, la curación, hasta la capacitación para el trabajo multi e interdisciplinario.

\section{DESARROLLO}

Venezuela está experimentando este mismo proceso de envejecimiento poblacional contando para el año 2018 con un porcentaje de personas de 60 años y más cercano al $10 \%$ y con una esperanza de vida de 77,18 para las mujeres y de 71,22 años para los hombres, según las proyecciones del Instituto Nacional de Estadística (INE:2011) encontrándose, según un informe presentado por el Centro Latinoamericano y Caribeño de Demografía (CELADE) - División de Población de la CEPAL-CELADE por lo que se halla entre uno de los primeros países que, junto con la Guayana Francesa, Costa Rica, Colombia presenta el mayor incremento de población adulta mayor de la región, con tasas superiores al 4\%, ubicándose entre el grupo de quince países de Latinoamérica que se encuentran en una transición moderada hacia un envejecimiento poblacional.

Ahora bien, las ideas anteriormente planteadas describen la situación demográfica Latinoamericana y venezolana de forma general; sin embargo como se ha señalado, el envejecimiento poblacional es la expresión de un proceso que experimentan los seres humanos, es por ello que así como envejecen las regiones, ciudades, los pueblos, en esa misma medida envejecen los individuos.

En tal sentido puede definirse el proceso de envejecimiento como "un proceso biofisiopsicológico, está constituido por una sucesión de modificaciones morfológicas, 


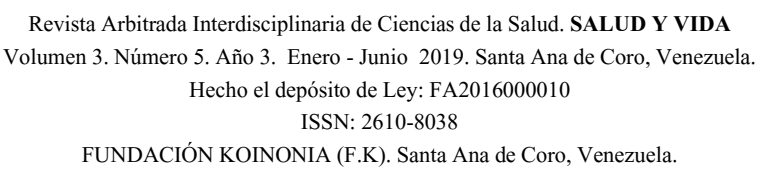

Maika Esther Bravo; Tibisay Lamus de Rodríguez

fisiológicas y psicológicas, de carácter irreversible, que inicia mucho antes que sus manifestaciones den al individuo el aspecto de viejo" (Langarica,1985:4) para Anzola y col., 1994:45) "es un proceso dinámico, irreversible, progresivo en el que intervienen múltiples factores biológicos, psicológicos y sociales." En ambas definiciones se evidencia la existencia de un proceso que conlleva a la etapa de la vejez en el que se producen modificaciones en el aspecto biológico de carácter irreversible asociados a un declive funcional y cambios psicológicos con implicaciones en los aspectos sociales. Es importante señalar en función de las ideas anteriores que, generalmente la vejez se caracteriza por la progresiva disminución de la funcionalidad, entendida como la pérdida de la capacidad de realizar por sí mismo y sin ayuda las actividades básicas, instrumentales y avanzadas de la vida diaria, el incremento en la probabilidad de sufrir múltiples enfermedades; y desde el punto de vista psico-social existe mayor probabilidad de experimentar sentimientos de soledad y depresión, baja autoestima, una merma en los recursos económicos, cambios en los roles sociales y, finalmente, la muerte en las edades avanzadas se debe principalmente a enfermedades crónico - degenerativas, con un proceso largo y costoso para la familia, pero también costoso institucionalmente por los requerimientos humanos y materiales necesarios para las acciones de curación y rehabilitación (Langarica:1985).

Todo lo antes planteado señala cómo las consecuencias del envejecimiento individual y por ende poblacional, impactan e impactarán a nivel general en las políticas de salud que deberán ser diseñadas para atender las necesidades crecientes de este grupo etario y a nivel institucional, indican la prioridad de diseñar acciones multi, interdisciplinarias e intersectoriales cuyo enfoque no sea exclusivamente psicológico, social o sanitario, sino que apunten hacia un enfoque de atención integral que se reflejen en las políticas sociales en materia de atención, en la búsqueda que más personas puedan lograr un envejecimiento saludable y activo; acciones que operen sobre la propia persona mayor vista integralmente y sobre el contexto en el cual ésta se encuentra inserto.

En tal sentido, para Anzola y col. 1994:XVI) desde el año 1982 ha venido funcionando un "Programa Regional de Salud de los ancianos" perteneciente a la Organización 
Panamericana de la Salud programa que ha estado realizando estudios, eventos científicos internacionales con el objeto de producir conocimiento científico en torno a la salud de las personas mayores. Una de sus conclusiones en torno a las políticas de atención al adulto mayor refiere :

se ha reconocido que las principales debilidades del desarrollo de programas coherentes para los ancianos son: a) el bajo nivel de conocimiento científico sobre el tema del envejecimiento y de la atención de los ancianos por parte de los profesionales vinculados al tema(...) como producto de la falta de incorporación del mismo en los planes de estudio de los centros de formación profesional, y b) la ausencia de políticas y planes nacionales, estatales y locales que estén orientados al apoyo solidario para el bienestar de un grupo que ha sido un factor indiscutible en el progreso social de los pueblos y que rechaza, abierta o secretamente, las acciones de beneficencia y humillación que mayormente reciben.

Desde la perspectiva anteriormente señalada, es importante considerar que todas las acciones que redunden a favor de la salud de la persona mayor en el mundo y particularmente en Venezuela, dependerán directamente de varios factores entre los cuales puede señalarse: los modelos económicos asumidos, los aspectos demográficos y sus demandas, el marco legal vigente a nivel nacional e internacional, la visión que los entes responsables del diseño de las políticas de salud posean en relación a la etapa de la vejez y de las formas cómo enfocarán sus políticas de atención.

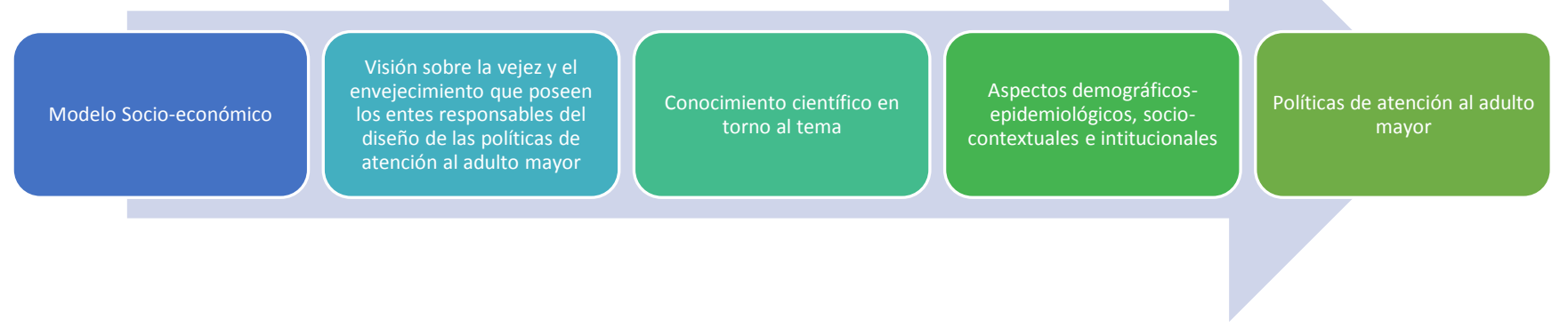




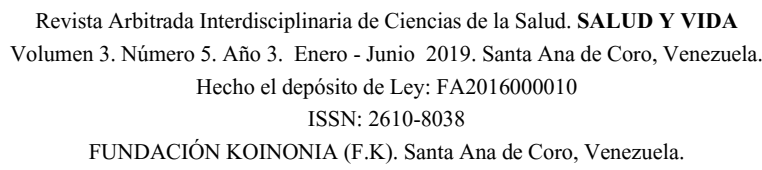

Maika Esther Bravo; Tibisay Lamus de Rodríguez

Es tal el impacto en las políticas sociales de la representación social que se tiene de la vejez por parte de los entes responsables, que la Organización de Naciones Unidas (ONU: 2008:75 ) en el documento "Guía para la Aplicación Nacional del Plan de Acción Internacional de Madrid sobre el Envejecimiento" se hace énfasis en las implicaciones y consecuencias para el bienestar de las personas de edad en todo el mundo; señalando que, "por un lado, las visiones sesgadas refuerzan la tendencia a excluir a la población de más edad de los procesos de adopción de decisiones sociales, económicas y políticas, al tiempo que pueden constituir la coartada tras la cual se les niegue otras oportunidades". Tales consideraciones se resumen en el siguiente texto:

En numerosos países y culturas, las personas de edad y el mismo envejecimiento acaso sean vistos de forma negativa toda vez que la participación de la mano de obra sea considerada la piedra angular del desarrollo social y económico. Donde las personas de edad son consideradas como un grupo que no participa directamente en procesos productivos, estas tal vez no sean vistas como miembros iguales de sus comunidades. En consecuencia, es posible que no disfruten de las mismas oportunidades de que disfrutan personas de otros grupos de edad, especialmente en casos en que las prioridades de las políticas públicas giren alrededor de las necesidades de la población trabajadora y no de todos los grupos sociales.

Esta representación social negativa en torno a la vejez y el proceso de envejecimiento condiciona la existencia no sólo el tipo de política destinada a la persona mayor, sino incluso incide en la asignación de recursos, el diseño de planes de formación destinada a los profesionales de atención directa y las oportunidades de empleo de éstas, la creación de instituciones destinadas al adulto mayor, hasta incluso la forma cómo son planteados los derechos en los instrumentos legales, entre otros aspectos.

Por otra parte, en relación a los aspectos demográficos y las políticas sociales, todos relacionados con los aspectos macro determinantes que inciden en el estado de salud de la persona mayor se tiene que, los entes responsables deben al momento de diseñar los planes y programas destinados a la persona mayor, tomar en cuenta las proyecciones epidemiológicas-demográficas de Venezuela emanadas de los censos poblacionales y los boletines epidemiológicos que de forma constante son ofrecidos por parte del Instituto 


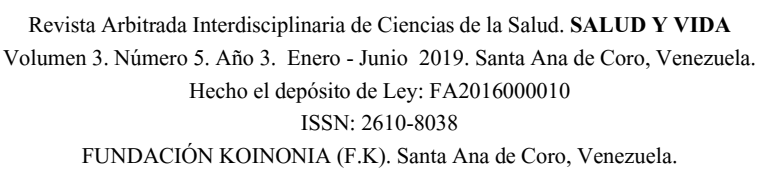

Maika Esther Bravo; Tibisay Lamus de Rodríguez

Nacional de Estadística, así como por parte del Ministerio con competencia en materia de Salud; estos datos aseguran que en el proceso de planificación de las políticas públicas de carácter social se aproximen lo más posible a la realidad del país, sin embargo según Reyes (2002):

La relativa juventud demográfica de Venezuela influye para que las diversas políticas y programas sociales se destinen mayoritariamente a los sectores jóvenes, relegando a la población longeva, de escaso peso demográfico. De hecho, existe una presencia muy débil de planes y políticas coherentes en favor de los ancianos, tanto por parte de las instituciones gubernamentales como por las organizaciones no gubernamentales; trayendo como consecuencia, la necesidad imperiosa y a corto plazo, de abrir un espacio político que permita otorgar una identidad definida a este grupo social, así como de suministrar los recursos necesarios para desarrollar programas viables y coherentes tendentes a satisfacer las urgentes necesidades de la población anciana.

Lo antes planteado permite ir perfilando una forma particular de entender el fin último las políticas sociales en Venezuela en relación a la atención de las personas mayores, centradas en abordar a la población joven, pero obviando el impacto demográfico que se ha venido avizorando a través de los censos poblacionales y que señalan la irreversible realidad de una Venezuela envejecida en tiempos cercanos y con políticas sociales de poco impacto en este grupo poblacional.

Etapas en el proceso de atención integral de las personas mayores en Venezuela Las primeras formas de atención al adulto mayor venezolano provinieron de las propias familias quienes, por ser un integrante del núcleo familiar, fuente de sabiduría, respeto y consideración, asumieron el cuidado en los momentos en los que las condiciones de salud o producto de las declinaciones normales del envejecimiento ameritaban la ayuda de otra persona. Posteriormente, las primeras formas de atención por parte del Estado, fueron las hechas por los Incas quienes, reconociendo el valor de sus ancianos, crearon formas de responsabilidad de sus gobernantes hacia sus mayores. 
Sin embargo, con el advenimiento de los procesos de la industrialización, la modernidad, sumado a la discriminación hacia los adultos mayores por razones de productividad y por lo tanto su exclusión y sometimiento a condiciones de extrema pobreza, enfermedades, abandono, el cuido de las personas mayores fueron asumidas por parte de personas con espíritu filantrópico y grupos religiosos, ofreciendo la satisfacción de las necesidades básicas para la subsistencia.

En el caso de Venezuela, la realidad en materia de atención no tuvo significativas diferencias de los países de Latinoamérica o Europa; se considera que el proceso de atención al adulto mayor venezolano inició con una primera etapa que las autoras denominarán De incipiente institucionalidad ( ver figura no2) en ella se identifican las acciones realizadas tanto por parte de la familia, como institución básica de atención informal ha sido la responsable del cuido de sus adultos mayores desde tiempos inmemoriales; así como se identificaron la existencia de organizaciones de tipo voluntariado( Cruz Roja, Damas Salesianas, entre otras organizaciones religiosas), las cuales desde sus posibilidades se advocaron a ofrecer los cuidados necesarios en los casos de indigencia, abandono o cualquier otra forma de vulnerabilidad social. Es decir que en Venezuela existió en esta primera etapa áreas sustantivas de atención: necesidades básicas y salud, sin institucionalidad estatal definida, sin objetivos claros y llevadas a cabo por personas sin preparación para atender las particularidades de la vejez.

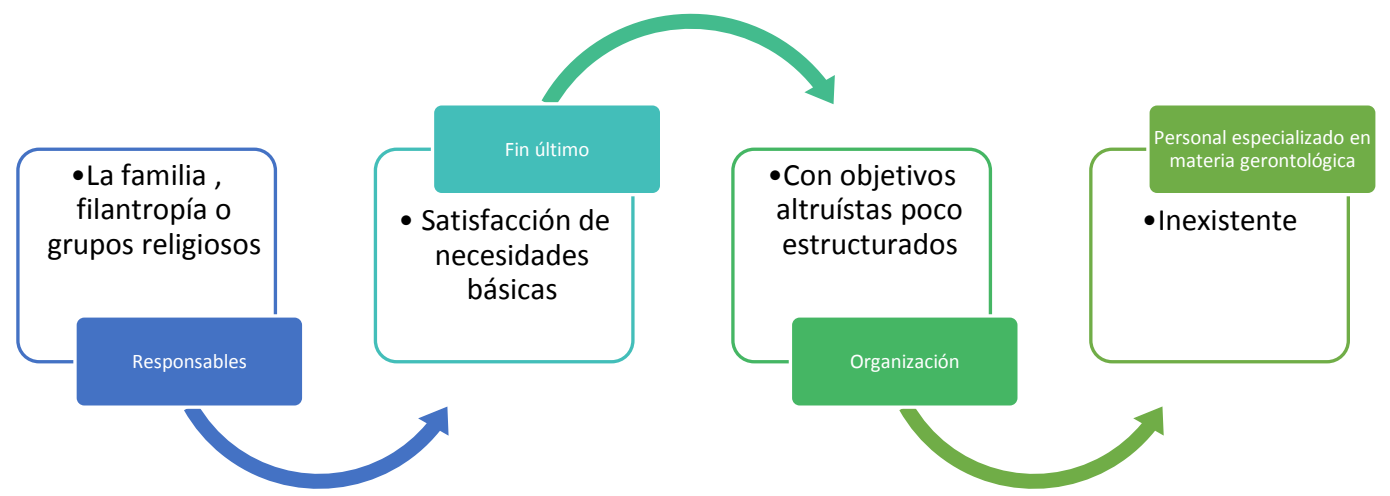

Figura2 Etapa de incipiente institucionalidad 


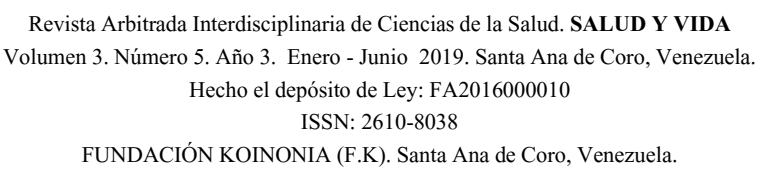

Maika Esther Bravo; Tibisay Lamus de Rodríguez

Ahora bien, posteriormente a esta primera etapa, comenzaron a surgir las políticas sociales e instituciones a favor de la tercera edad en Venezuela; políticas que confluyeron con modelos económicos, condiciones sociales y un marco legal donde la seguridad social comenzaba instaurarse separada de la asistencia social, con lo cual se configuraron formas de atención al adulto mayor que aún hasta hoy a nivel del Estado como generador de planes y políticas públicas, no logran desprenderse de las representaciones sociales negativas asociadas a esta etapa.

Puede decirse entonces que la atención del adulto mayor continúa con una segunda fase denominada por las autoras como Etapa de inicio de la institucionalidad de la atención al adulto mayor (ver figura no3), donde se desarrollan programas sociales bajo un modelo económico-social de corte universal-asistencialista, que respondieron al modelo conservador caracterizado por políticas sociales diferenciadas para CEPAL (2004) en este modelo los programas sociales en países en desarrollo se caracterizan por la inversión en políticas masivas y gratuitas como educación, salud y saneamiento ambiental destinadas a toda la población; b) de aseguramiento contributivo para los trabajadores ante contingencias que escapan a su control: desempleo, incapacidad, vejez, enfermedad; en Venezuela fue materializado a través de la creación y expansión del Seguro Social Obligatorio instrumentalizado a través del Instituto Central de Seguridad Social (posteriormente Instituto Venezolano de los Seguros Sociales) entre 1940-1944 con bajas coberturas de aseguramiento en asistencia médica, debido a su concentración en los centros industriales del país; c) de protección y/o asistencia para poblaciones sin capacidad de contribuir, porque pertenecen a familias donde falta el apoyo económico o existen ciertas fragilidades que ponen en riesgo o vulnerabilidad a las personas.

En relación a la asistencia social, se creó como institución destinada a las personas de la tercera edad el Patronato Nacional de Ancianos e Inválidos (PANAI) en 1949 según gaceta oficial n 23.089, adscrito al Ministerio de Sanidad y Asistencia Social, según la cual su objetivo era "la atención de los ancianos y al inválido, protección que se daría a través de instituciones propias a ese fin o en su ambiente familiar". Esta atención estaba 


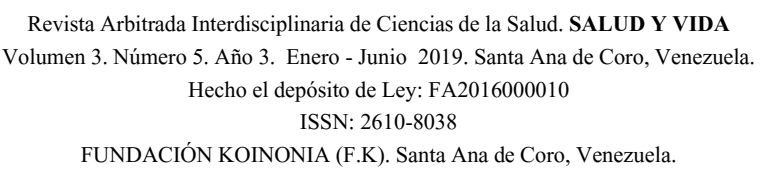

Maika Esther Bravo; Tibisay Lamus de Rodríguez

destinada a personas de 65 años y más, sin recursos económicos, sin patologías infectocontagiosas, o trastornos mentales y no asegurados por parte del Instituto Venezolano de los Seguros Sociales (Aponte, 1978:63).

Esta misma autora señala que:

En los comienzos, sus acciones se caracterizan por una fundamentación empírica y benéfica como consecuencia lógica de no contar con objetivos claros y definidos, de acuerdo a programas específicos. Por otra parte, entre sus primeros directivos se contaron con filántropos preocupados por la problemática social pero desprovistos de enfoques tecnológicos... es después de los años 1958-60 que al frente de la institución estuviera el Dr. Joaquín Quintero pionero de la geriatría en Venezuela, quien le imprimió carácter científico a la atención y emprendió la reorganización institucional(Idem:64)

Una de las consideraciones importantes de la mencionada autora en relación a el PANAI es que sólo es después de 1969 toma el impulso programático y reorganizativo con "una verdadera orientación gerontológica, dándole el verdadero apoyo a los programas de atención social a través del Departamento de Trabajo Social, con los enfoque técnicos requeridos"(Op. Cit. pág. 65); en ella se llevaron a cabo subprogramas socio-médicos (de carácter curativo a residentes y preventivo a nivel ambulatorio), bienestar familiar y sociocultural en la búsqueda la calidad de vida del adulto mayor y su grupo familiar. Sus objetivos posteriores fueron ampliar la cobertura a través de la atención médica social integral no solo a nivel institucional sino ambulatorio en cada una de las regiones. Esta institución no contaba con personal especializado para ofrecer atención bio-psico-social a las personas destinatarias, con lo cual se redujo su capacidad para mejorar su calidad de vida, reduciéndola a la satisfacción de necesidades básicas: abrigo, vivienda, alimentación.

En otras palabras, en una evaluación realizada al PANAI en 1972 a sus instituciones se encontró que poseían limitaciones y problemas importantes en cuanto a la calidad de atención ofrecida en virtud de: la accesibilidad(algunas se encontraban fuera del perímetro de la ciudad; en cuanto al destino original de las residencias, de éstas el $50 \%$ fueron de orientación gerontológica, el resto fueron destinadas originalmente a ser una 


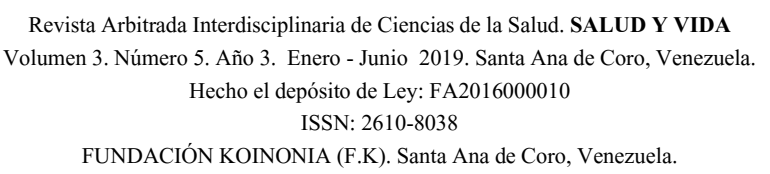

Maika Esther Bravo; Tibisay Lamus de Rodríguez

vivienda familiar, hasta un servicio de estacionamiento y línea de autobuses; en cuanto a sus programas, sus fines fueron inadecuados en virtud de la heterogeneidad de la población atendida (ancianos sanos con invalidez, enfermos crónicos y algunos trastornos graves conductuales). Señala la misma autora que el contar con una población tan desigual, ameritaba la existencia de programas específicos que no se implementaron. (Aponte: 1978);

Del mismo modo se encontró en el análisis hecho por la autora que la interacción entre el personal que labora en las instituciones del PANAI y los residentes era baja y poco satisfactoria, esta autora señala que pudo ser debido a que el personal no era seleccionado vocacionalmente, era carente de orientación sobre las características de este grupo etario, la capacidad de tolerancia y comprensión necesaria; otro rasgo importante para considerar es que la autora señala que, en cuanto al nivel de instrucción, un 11,4 de los obreros eran analfabetas, el 32,6\% del personal solo sabían leer y escribir; el 1,5\%poseía secundaria completa, un 0,7\% alcanzó la educación superior y 0,3\% otros estudios(IDEM: 1978). 

Hecho el depósito de Ley: FA2016000010

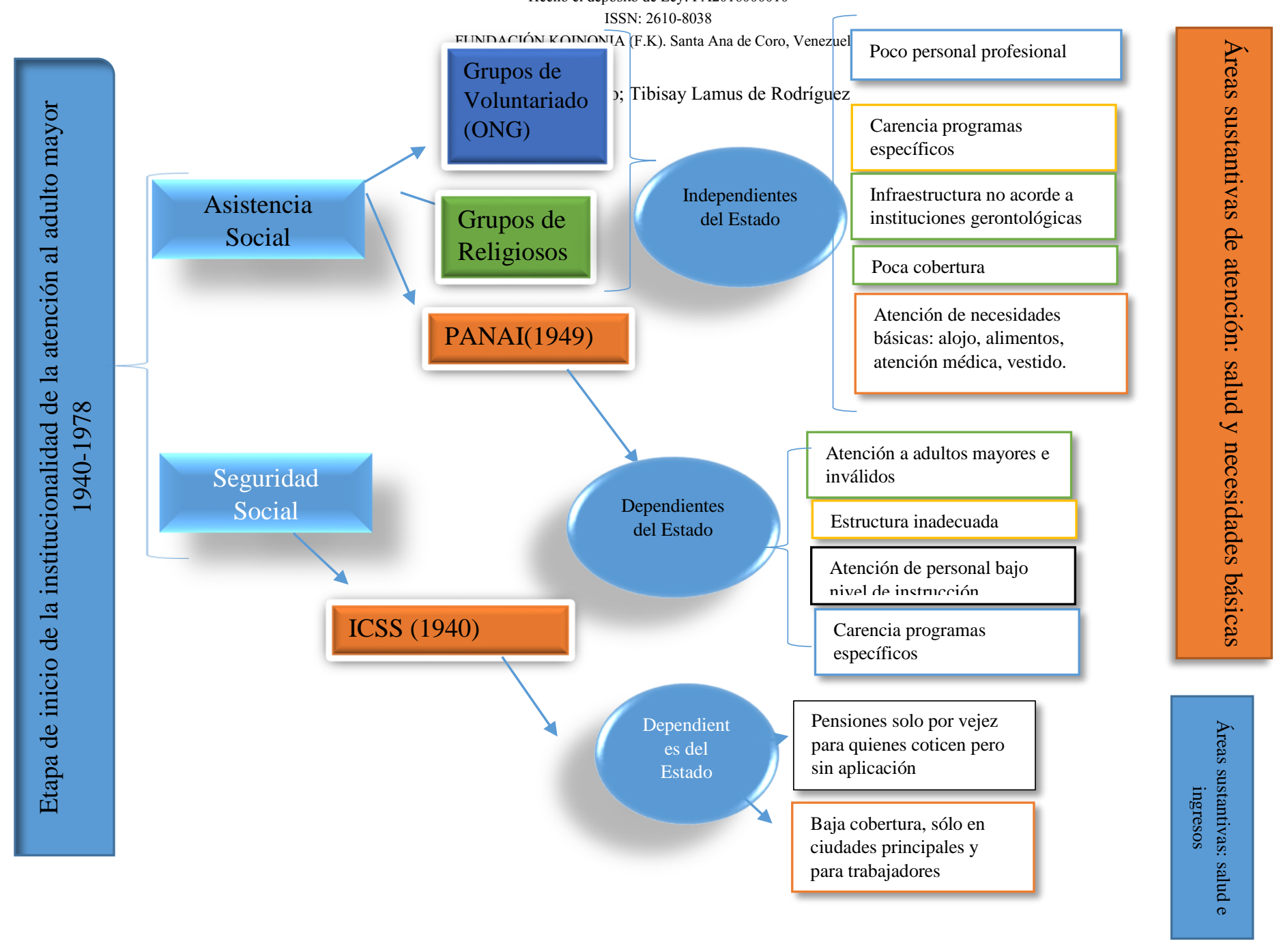

Posterior a esta Etapa de inicio de la institucionalidad de la atención al adulto mayor, se desarrolló una etapa denominada también por las autoras como de Incursión de la gerontología a la Atención del adulto mayor(ver figura no4), esta etapa se desarrolló bajo un modelo económico selectivo-compensatorio de enfoque (neo) liberal que incidió en las políticas sociales destinadas a las personas mayores entre los años 80 hasta finales del 90. Comprender el contexto socio-histórico de esta etapa permitirá entender el tipo de políticas destinadas a la atención del adulto mayor.

Durante este período, desde el punto de vista político-económico, la crisis que se generó a nivel mundial en la esfera económica y que repercutió directamente en Venezuela al disminuir los precios del petróleo y por tanto los ingresos económicos, imposibilitaron el 


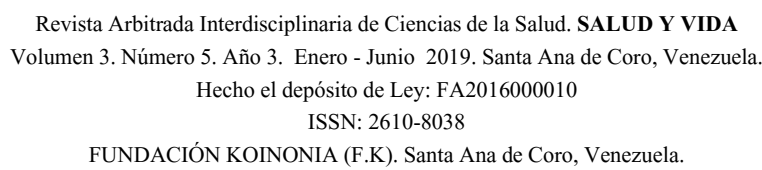

cumplimiento de las responsabilidades asumidas con el modelo anterior. Como consecuencia el Estado de Bienestar tuvo que atribuirle responsabilidades compartidas a la sociedad en materia de educación, en materia de salud y sobre todo en materia de atención al adulto mayor. Es por ello que sólo se dedicó a proteger a la población vulnerable a través de programas sociales focalizados, tratando de atacar la pobreza extrema. Todos los programas diseñados poseían corte asistencialista fundamentados en una imagen de los destinatarios de carga, debilidad y necesidad.

En materia de salud, se evidenció un proceso de tanto de segmentación como de dispersión de las instituciones y programas de salud, debido por una parte, a la crisis económica mencionada y por lo tanto las dificultades del Estado de satisfacer las demandas del Sistema Público Nacional de Salud. Por otra parte, las dificultades administrativas por parte del IVSS (por bajos presupuestos) impidieron ofrecer la atención de salud de calidad a los asegurados que aumentaron en número al incluirse no sólo a los obreros que habían sido protegidos en los años 40, sino la ampliación a las familias de éstos y posteriormente la inclusión del sector privado, el cual había estado desprotegido.

Esta dificultad presupuestaria, la baja calidad de la atención, las dificultades de atención en la red ambulatoria incidieron en la creación de más de 100 subsistemas de aseguramiento en pensiones y salud: Alcaldías, Gobernaciones, Ministerios de Defensa, Educación, la Contraloría a nivel de los estados, entre otros, en búsqueda de garantizar la salud a aquellos que pudiesen cubrir los gastos, valiéndose de la descentralización como estrategia para su consolidación, en detrimento de la universalización de la salud. En relación a la seguridad social ofrecida por parte del IVSS se evidenció una progresiva extensión en pensiones por vejez, invalidez y sobrevivientes, consagradas en ley a partir de enero de 1947 pero que se hicieron efectivas a partir de 1971, a pesar que el Instituto Central de los Seguros Sociales fue en creado en 1940; en cuanto a la asistencia social, los organismos gubernamentales (gobernaciones y alcaldías) se centraron en ofrecer beneficios a sus empleados de carácter dinerario una vez cesaran de sus actividades laborales al cumplir 25 años de servicio, cuyo monto dependía del cargo, nivel de 


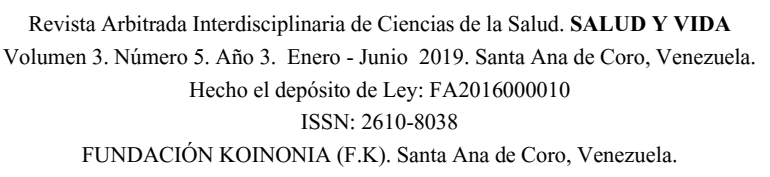

Maika Esther Bravo; Tibisay Lamus de Rodríguez

instrucción, entre otros requisitos; así como el otorgamiento de ayudas económicas a personas en estado de necesidad con montos que se acercaban al $60 \%$ del salario mínimo, con lo cual sólo se benefició una parte de la población.

Siguiendo el orden de las ideas anteriores, en materia institucional de atención al adulto mayor el PANAI paso a denominarse para el año 1978 Instituto Nacional de Geriatría y Gerontología (INAGER), institución que tomó las riendas de la atención de la población mayor, separándose de la atención de otros grupos vulnerables con necesidades diferentes a la de las personas de la tercera edad. Con esta disposición se le ofrece una atención a los adultos mayores a través de la modalidad de la atención cerrada a través de las Unidades Geriátricas en las cuales se les ofrecía a sus residentes atención no sólo de sus necesidades básicas, sino programas de terapia ocupacional, fisiatría, actividades recreativas y deportivas acorde a las particularidades de este grupo etario Para Reyes (2003) la situación en cuanto a la atención de esta institución entre 19702003 no permitió atender a una población cada vez más numerosa y con necesidades específicas, es por ello que considera que "apenas se atiende a un 4 a $6 \%$ de ancianos de escasos recursos económicos, sin que se haya desarrollado ni fortalecido una estructura gerencial que le permita al INAGER prestar un mejor servicio y asistencia social a su población objetivo." Esto debido a que la atención ofrecida a las personas mayores estuvieron planificadas y desarrolladas en un primer momento, por personas con poca preparación para atender a este grupo poblacional, generalmente médicos, trabajadores sociales y psicólogos, cada uno con su visión profesional y sus propios objetivos inclinando la balanza en cuanto a los fines de los programas en función de sus áreas de conocimiento.

Otro de los aspectos resaltantes es que para el año 1986 incursiona en el ámbito universitario la carrera de Gerontología, de la mano de la Universidad Nacional Experimental Francisco de Miranda, egresando para 1989 la primera cohorte de Técnicos Superiores Universitarios en esta área de conocimientos. Todos estos profesionales fueron absorbidos tanto por la propia universidad y por el recién creado INAGER, con lo 
Revista Arbitrada Interdisciplinaria de Ciencias de la Salud. SALUD Y VIDA

Volumen 3. Número 5. Año 3. Enero - Junio 2019. Santa Ana de Coro, Venezuela. Hecho el depósito de Ley: FA2016000010

ISSN: 2610-8038

FUNDACIÓN KOINONIA (F.K). Santa Ana de Coro, Venezuela.

Maika Esther Bravo; Tibisay Lamus de Rodríguez

cual se comienza a avizorar en la etapa subsiguiente, un cambio en las políticas de atención de esta institución.

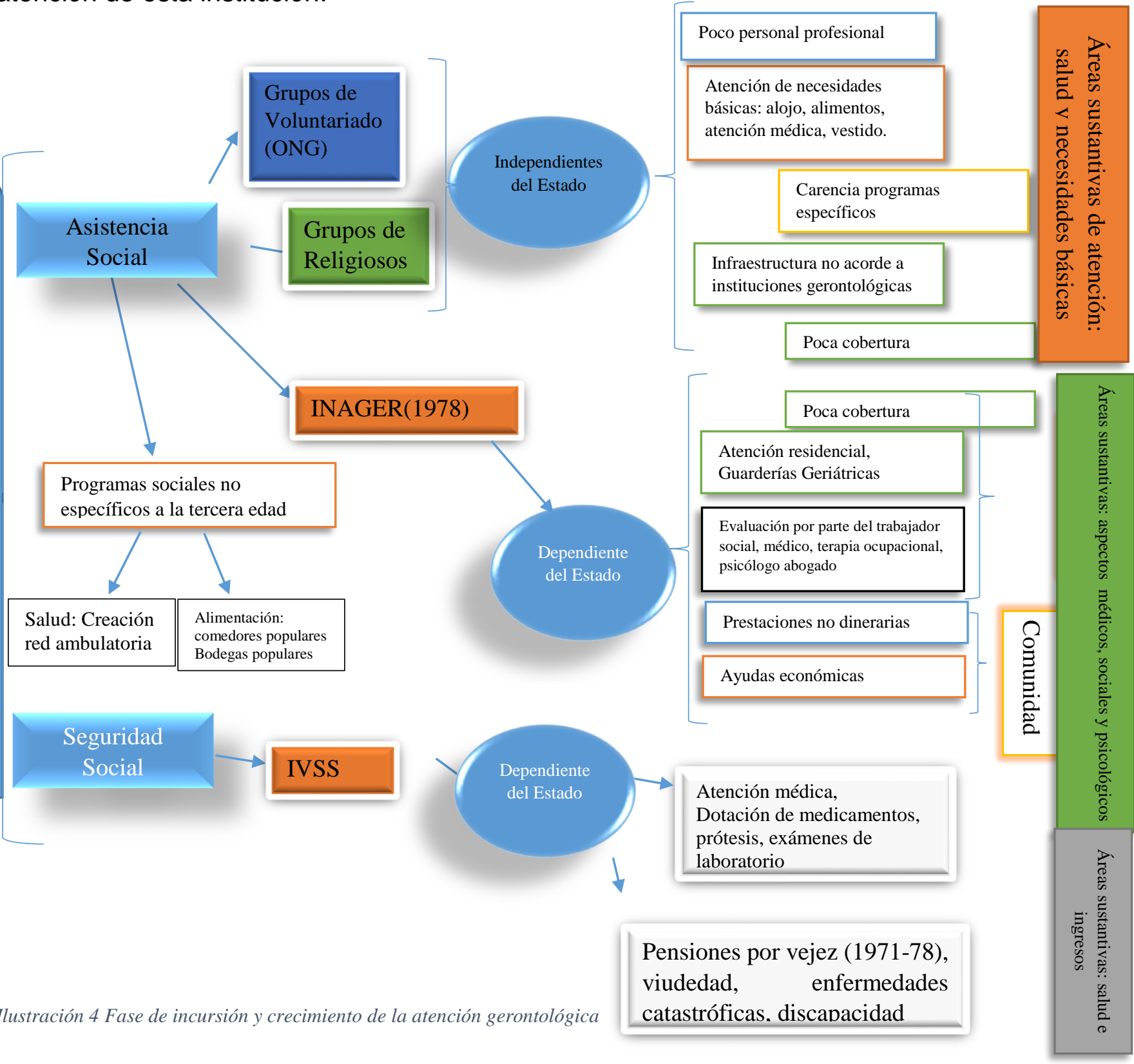

Ahora bien, desde el año 1990 a la actualidad en Venezuela en materia de atención gerontológica se puede mencionar que se desarrolla la etapa que las autoras denominaron como de la ampliación y consolidación de la atención gerontológica; esta denominación se debe a la incursión de los gerontólogos en diferentes espacios: 


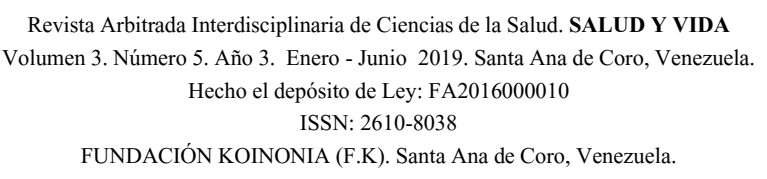

Maika Esther Bravo; Tibisay Lamus de Rodríguez

instituciones cerradas, instituciones pertenecientes al Sistema Público Nacional de Salud, Fuerzas Armadas, entre otros.

Esta etapa se desarrolla en el marco de un contexto económico-político donde se retoman algunos aspectos de ambos modelos en materia de políticas sociales presentados en las etapas anteriores, encontrándose que la política social (más allá de la tradicional), al menos hasta el presente, ha estado orientada a la atención de los síntomas de la pobreza, de modo que se trata de una política asistencialista y poco específica en materia de grupos etarios, con un criterio de reparto de bienes o servicios de manera indiscriminada, y siempre insuficiente, desconociendo las diferencias regionales, entre lo urbano y lo rural, e incluso sin considerar la posible heterogeneidad existente entre los grupos a quienes atiende.

En materia de atención al adulto mayor a través de la Seguridad Social, se continúa con el otorgamiento de pensiones por vejez a través del IVSS cuyo monto se iguala al salario mínimo, incluyéndose también contingencias como enfermedades catastróficas, viudedad y discapacidad. Se amplía a partir del año 2005 las personas que pueden acceder a las pensiones por vejez: amas de casa, trabajadores del volante, cultores, pescadores, artesanos, todos estos grupos de la sociedad estaban desprotegidos al no haber accedido a un empleo formal que le permitiesen cancelar las 750 cotizaciones necesarias para poder asignarle una pensión.

En cuanto a la asistencia social, a partir del año 2005 se busca ampliar la cobertura de las asignaciones ofrecidas por el Estado venezolano, es así como se le eliminan las atribuciones que poseía el INAGER de ofrecer ayudas económicas a las personas en estado de necesidad, para que fueran asignadas al IVSS y elevadas su cuantía; este proceso se llevó a cabo a través de la estrategia denominada "Misión Amor Mayor" la cual permite agilizar los trámites para su otorgamiento y ampliar la cobertura. Al mismo tiempo se crearon otras misiones sociales con impacto en la población mayor; Misión Robinson I y II, (de carácter educativo) Misión José Gregorio Hernández y Misión Milagro (abordan aspectos relativos a la salud). Aunque son buenos intentos para atender las necesidades de los adultos mayores, no poseen un impacto estructural que permitan 
que más personas adultas mayores alcancen un envejecimiento saludable, activo y autónomo.

Entre el año 1990 y el año 2005, se hizo un viraje en las políticas de atención institucional por parte del INAGER, en virtud de la incorporación de nuevas cohortes de profesionales de la gerontología, con ello se pasó de la sola atención residencial a la creación de programas como las Guarderías Geriátricas(dentro de la misma institución) a través de la cual se ofrecía atención por un tiempo determinado durante el día( 8:00 am a 4:00 pm) que posteriormente se denominados Centros de Atención Diaria, en virtud de con los cambios de paradigma a nivel mundial en materia de envejecimiento y vejez instrumentalizados en los Planes de Acción Internacional (Viena y Madrid), estas guarderías ofrecían los mismos beneficios que a los residentes. Una de las consideraciones de la investigadora es que tanto el término Unidad Geriátrica como el de Guardería Geriátrica, son denominaciones con una carga de significado negativo que asocia a la vejez con la enfermedad, dependencia, niñez. Estas denominaciones a pesar de ser modificadas posteriormente, aún permanece en la representaciones sociales que maneja la sociedad cuando se trata de referirse a estas instituciones

En cuanto a la atención cerrada, al incorporarse al equipo de salud el profesional de la gerontología, el abordaje que se lleva a cabo pasó a ser integral en la búsqueda de no sólo ofrecer atención médica, valoración social por parte de los trabajadores sociales, apoyo jurídico, laborterapia y rehabilitación, sino que también se incluye una serie de actividades de carácter formativo destinadas al fomento del autocuidado, la formación del personal de las instituciones en materia gerontológica. Se incluyen programas a nivel nacional (según particularidades) como las Gerogranjas, los Hospitales de Día(Aragua), visitas domiciliarias, entre otros programas.

A partir del año 2005, según la Ley de Servicios Sociales(2005) el INAGER pasó a denominarse Instituto Nacional de Servicios Sociales dependiente del Ministerio del Poder Popular para las Comunas y Protección Social según Gaceta Oficial № 38.270 del 12 de septiembre de 2005 , institución destinada a ofrecer atención integral a todas las personas adultas mayores y otras categorías de personas entendidas como "Personas 


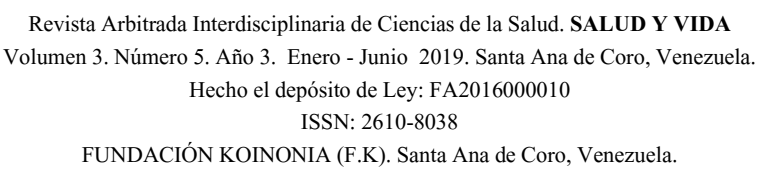

Maika Esther Bravo; Tibisay Lamus de Rodríguez

naturales menores de sesenta años de edad, con ausencia de capacidad contributiva para cotizar al Sistema de Seguridad Social, que se encuentren en estado de necesidad y, no están amparadas por otras leyes, instituciones y programas" (artículo 7 parágrafo 2). Se entiende a las personas en estado de necesidad como aquellas que estén en situación de desprotección económica, personal, familiar o social, que desencadena necesidades sociales previstas y tipificadas por esta Ley como merecedoras de protección temporal o permanente(Artículo 7 parágrafo 3) y les garantiza según el Artículo 9. Ios derechos humanos sin discriminación, los derechos de carácter civil, su nacionalidad y ciudadanía, los derechos políticos, sociales, de la familia, culturales, educativos, económicos, ambientales y de los pueblos.

El marco legal mencionado anteriormente permite afirmar que en cuanto a los aspectos institucionales, Venezuela retomó desde ese momento, los objetivos del PANAI dejando de ofrecer atención específica a las personas mayores, ampliando su cobertura a otras categorías de personas, pudiendo convertirse en un retroceso organizativamente hablando. Para la época el INASS se hizo cargo de albergar a personas indigentes, en estado de abandono, con problemas de drogo-dependencia, implementando programas de la Misión Negra Hipólita; aunque posteriormente se hicieron reestructuraciones que llevaron a que se separaran institucionalmente ambos programas; sin embargo, la ley sigue amparando a las personas mayores y las otras categorías de personas, con lo cual se merma la atención particular que cada grupo poblacional merece.

Ahora bien, en materia de atención abierta, además de los Centros de Atención Diaria(antiguamente Guarderías geriátricas), se crearon nuevas formas de atención a nivel comunitario; es así como particularmente en el estado Falcón, se creó en 1995 un Programa de Atención Integral destinado a los adultos mayores del municipio Miranda, diseñado por tres gerontólogos egresadas en el año 1994, dentro de las cuales se encuentra la investigadora, lo que facilita la descripción de esta forma de atención.

Este programa fue innovador por tres aspectos: fue el primero destinado a la población en las comunidades a nivel del estado y nacionalmente al ser visto como modelo de atención comunitaria; buscaba ofrecer una atención bio-psico-social a las personas 


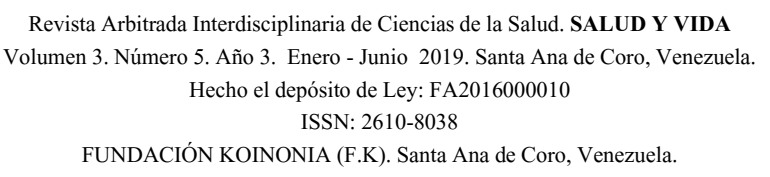

Maika Esther Bravo; Tibisay Lamus de Rodríguez

mayores y por último, se creó un Departamento Municipal de Atención Gerontológica, con su estructura organizativa. Fue presentado ante la Cámara Municipal y adscrito a la Dirección de Salud de la Alcaldía de Miranda a cargo del Dr. Ignacio Petit, teniendo como objetivo atender a la población mayor de las comunidades de la Urb. Cruz Verde, San Nicolás y Pantano Abajo, a través de la realización de subprogramas como: consulta gerontológica (el gerontólogo participaba en la consulta llevada a cabo por el médico, hacía seguimiento al cumplimiento del tratamiento, se gestionaba las consultas especializadas y la dotación de medicamentos. Este proceso de acompañamiento permitió ofrecer orientación también al núcleo familiar sobre el estado de salud de la persona mayor en función de garantizar el apoyo necesario.

Por otra parte, se desarrolló el sub-programa recreativo, deportivo y cultural y educación gerontológica continua y formación gerontológica (destinada a los trabajadores de los ambulatorios, no sólo donde se desarrollaban las actividades, sino también a los pertenecientes al municipio). Para organizar las actividades se crearon los primeros círculos de adultos mayores (denominación que se mantiene actualmente) los cuales desarrollaban sus actividades en el ambulatorio de los sectores mencionados, en un horario acordado entre gerontólogo-adulto mayor

Después del año 2000 el Sistema Público Nacional de Salud a nivel regional y posteriormente a nivel nacional, comienza a absorber algunos profesionales de la gerontología a través de los Sistemas Locales de Salud ( SILOS), se crean las Coordinaciones Regionales de gerontología y con ello se establece una estructura organizativa que busca realizar acciones sistemáticamente organizadas y bajo los lineamientos del Ministerio del Poder Popular para la Salud.

\section{CONSIDERACIONES FINALES}

Las consideraciones expuestas evidencian la forma como fue manejada la atención de la persona mayor por parte de las políticas de Estado desde sus inicios, la carencia de fundamentos teóricos producto de una incipiente investigación en materia de envejecimiento, escasa infraestructura, recursos económicos insuficientes, la centralidad 


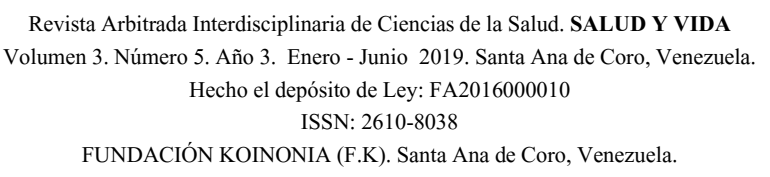

Maika Esther Bravo; Tibisay Lamus de Rodríguez

en la satisfacción de necesidades básicas y la falta de personal con conocimiento específico sobre la vejez y el envejecimiento, llevaron a ofrecer atención de carácter asistencialista enfocada en una imagen de carencia, debilidad, necesidad.

Sin embargo, de forma progresiva, a través de la incorporación del profesional de la gerontología en los ámbitos comunitarios ha ido incidiendo en la modificación de las políticas de atención a nivel comunitario e institucional, pero las políticas sociales destinadas a este grupo poblacional no han seguido los mismos pasos, manteniendo su carácter asistencialista, de atención focalizada que debe sobreponerse y dar respuesta a las demandas epidemiológicas y sociales presentes y futuras.

Es importante considerar que, para ofrecer atención integral al adulto mayor tal como lo establece el marco legal venezolano, es necesario integrar un conjunto multidisciplinario de visiones con el objeto de abarcar la multidimensionalidad del ser humano; es por ello que toda política de atención a este grupo etario debe contener elementos que permitan abordar los aspectos bio-psico-sociales y espirituales de la persona mayor evitando con ello la segmentación en la atención.

Lo anterior implica que los encargados de generar políticas sociales destinadas a las personas mayores en primera instancia deberían contar con un equipo de trabajo conformado por médicos, psicólogos, trabajadores sociales, economistas, sociólogos y gerontólogos que ofrezcan una visión general de la etapa de la vejez y el envejecimiento; así como partir de diagnósticos de la situación real de las personas mayores en Venezuela, lo que garantizaría que puedan satisfacerse las necesidades prioritarias de esta población que en un futuro cercano constituirán cercano a un $11 \%$ de la población total.

Todo lo antes planteado ha permitido afirmar que aunque se ha avanzado en materia de atención al adulto mayor, aún queda camino por construir, aspectos organizativos que alcanzar, políticas de atención que repensar, de tal forma que pueda lograrse una verdadera atención integral a la persona mayor. Esta ardua tarea no es solo responsabilidad de los profesionales de la gerontología, sino que también deben integrarse desde una visión amplia y sin estereotipos, una multiplicidad de profesiones 


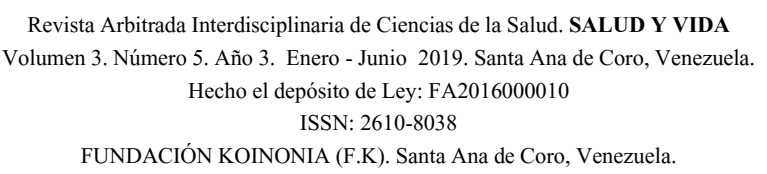

Maika Esther Bravo; Tibisay Lamus de Rodríguez

que contribuyan a que esta sociedad pueda considerarse para todas las edades y que más personas puedan alcanzar un envejecimiento saludable y exitoso.

\section{REFERENCIAS CONSULTADAS}

1. Anzola Elías, Galinsky David, Morales Fernando, Salas Aquiles, Sánchez Melba La atención de los ancianos un desafío para los años noventa. Organización Panamericana de la Salud (1994).

2. Aponte Margarita El anciano en la Sociedad venezolana 1era edición Caracas Universidad Central de Venezuela. Facultad de Ciencias Económicas y Sociales. División de Publicaciones. (1978).

3. Centro Latinoamericano y Caribeño de Demografía (CELADE) - División de Población de la CEPAL,(2009) "Estimaciones y proyecciones de población" [en línea] www.eclac.cl/celade/proyecciones/basedatos BD.htm (consultado 16 febrero 2019).

4. CELADE-División de población de la CEPAL (2006) L. Manual de indicadores de calidad de vida en la vejez.LCW-113 diciembre de 2006[en línea] www.eclac.cl/celade/proyecciones/basedatos BD.htm (consultado 16 febrero 2019).

5. CEPAL (Comisión Económica para América Latina y el Caribe) 2004), Panorama social de América Latina 2004 (LC/G.2259-P/E), Santiago de Chile. Publicación de las Naciones Unidas. [en línea] www.eclac.cl/celade/proyecciones/basedatos BD.htm (consultado 25 febrero 2019)

6. Instituto Nacional de Estadística (2011) Primeros resultados del Censo 2011. [en línea]: http://www.ine.gov.ve/CENSO2011/.(consultado 01 marzo 2019).

7. Langarica Salazar Raque-(1985). Gerontología y geriatría. Editorial Interamericana. México.

8. Pérez Fernández, R (1996) "Tiempos en el tiempo: Notas sobre el proceso de envejecimiento, la temporalidad y el cuerpo". En: Universidad de la República, Facultad de Psicología (1996) Historia, Violencia y Subjetividad: III Jornadas de Psicología Universitaria. Montevideo: Multiplicidades, pp.. 150-156. [en línea]: http://www.psiconet.com/tiempo/. (consultado 01 marzo 2019) 


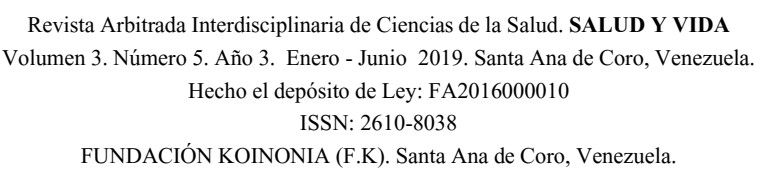

Maika Esther Bravo; Tibisay Lamus de Rodríguez

9. República Bolivariana de Venezuela Ministerio del Poder Popular para las Comunas y Protección Social Instituto Nacional de Servicios Sociales INASS Ley de Servicios Sociales(2005) Gaceta Oficial №38.270 12 de septiembre de 2005.

10. Organización de Naciones Unidas(2008) "Guía para la Aplicación Nacional del Plan de Acción Internacional de Madrid sobre el Envejecimiento.

11. Reyes, L (2003). Investigación Gerontológica y Políticas Sociales de Atención Integral al Adulto Mayor en Venezuela. Tesis Doctoral publicada. Doctorado en Ciencias: Investigación. Universidad Dr. Rafael Belloso Chacín. Maracaibo, estado Zulia, Venezuela.

12. SÁNCHEZ C (2000), Gerontología social, Buenos Aires Argentina: Espacio, 24187.

(C2019 por los autores. Este artículo es de acceso abierto y distribuido según los términos y condiciones de la licencia Creative Commons Atribución-NoComercial-Compartirlgual 4.0 Internacional (CC BY-NC-

SA 4.0) (https://creativecommons.org/licenses/by-nc-sa/4.0/). 